\title{
Performance Evaluation of Photonic Networks under Dynamic Traffic Conditions
}

\author{
Jan Späth and Stefan Bodamer \\ University of Stuttgart \\ Institute of Communication Networks and Computer Engineering \\ Pfaffenwaldring 47, D-70569 Stuttgart, Germany \\ Tel.: +497116857990, Fax: +497116857983 \\ E-Mail: \{spaeth, bodamer\}@ind.uni-stuttgart.de
}

\begin{abstract}
This paper focuses on two aspects of photonic network traffic performance investigation. Two different modelling approaches for the evaluation of network nodes under dynamic traffic conditions are described. This allows to evaluate how suitable different node architectures are for networks carrying dynamic traffic. Furthermore, routing strategies are becoming more important with increasing dynamic. A lot of work reported in literature deals with "wavelength routing". In most cases however, this means static wavelength assignment to routes and not dynamical routing of random wavelength path requests. This paper now considers dynamic traffic aspects of wavelength routing where many analogies to classical routing strategies can be observed. Therefore, a short overview of routing methods is given before we describe our modelling approach and present some case studies and results.
\end{abstract}

Keywords

Photonic transport network, WDM, performance evaluation, dynamic traffic

The original version of this chapter was revised: The copyright line was incorrect. This has been corrected. The Erratum to this chapter is available at DOI: 10.1007/978-0-387-35398-2_19 


\section{INTRODUCTION}

Photonic technology is able to handle the expected future traffic growth. Although it is not clear how future networks will look in detail, a promising solution is to enhance present networks with a transport layer based on WDM transmission technology and network nodes with cross-connect (CC) functionality. A lot of technological as well as theoretical research has been done. One big field is optimised network planning with the goal of minimising the required network resources. This is usually done for static traffic requirements using more or less complicated optimization strategies. However, real network operation will not only see static traffic requirements but also dynamic behaviour basically due to two reasons: unexpected network failures and random traffic changes. The latter is caused by the inherent randomness of user behaviour and environmental changes. Therefore the consideration of traffic behaviour plays a central role in all network planning and evaluation methods applied in the past.

In future photonic networks, the dynamic will further increase. Although many details are still not clear and heavily discussed, the following scenario seems to be realistic:

- Today, we see mainly static point-to-point WDM channels, soon supported by protection paths.

- The next step will see wavelength paths passing multiple nodes. First, these paths will be realised via fixed nodes like ADMs, then reconfiguration possibilities will be added including protection schemes.

- Further increase of flexibility could then be achieved by using optical CCs and the possibility for the network management to establish wavelength channels dynamically to adapt to requirement changes.

- In the long-term, at least partly control of channels by user signalling could also be possible.

Since in the foreseeable future the optical layer will serve as a transport layer, the dynamic in this layer is determined by the aggregation of many user traffic streams and therefore not easy to predict. Basically, the dynamic will decrease when going from the user to the transport layer, the granularity however will increase. Therefore, a dynamic consideration of the transport layer is important due to the huge impact of blocking effects in this layer.

\section{ROUTING METHODS FOR CIRCUIT-SWITCHED PHOTONIC NETWORKS}

Photonic networks will be based on circuit-switching (CS) in the foreseeable future. Various routing methods exist for CS networks and also several classification schemes can be applied. Some classification criteria applicable for CS as well as for packet switched (PS) networks are listed below. An overview of these and more routing strategies can be found in [1]. 
- adaptive / non-adaptive: Adaptive routing methods consider the network situation (traffic load, equipment status, ...) at the moment of a call request whereas for non-adaptive methods routing decisions are made independent of the present network situation.

- source routing / hop-by-hop routing: When using source routing schemes, the route is determined at the source node of a call request. If multiple alternatives are available, this node is also responsible for the choice between them. Hop-byhop routing uses step-wise routing by forwarding the routing control from node to node until the end node is found. The great variety of rules for the forwarding process leads to many different routing strategies.

- central / distributed / isolated: All central schemes use a central network control instance (often called Routing Control Centre, RCC) for the routing decisions whereas for isolated schemes each network node makes the decisions based on its own information base without communicating with other nodes. Distributed schemes make use of active information exchange between several nodes.

- hierarchical / non-hierarchical: Hierarchical routing looks for routes using the network hierarchy as guideline (e.g. routing in classical telephone networks) whereas non-hierarchical strategies use paths independent of hierarchical properties of the network topology (an example is routing in the internet).

For routing in photonic networks, many analogies exist to classical networks. By comparing the wavelength domain of a photonic network with the time domain of a classical network, one can state the following. From a functional point of view, photonic networks which are fully equipped with wavelength converters can be compared with classical TDM networks with time-slot interchange (fully equipped means, that in every network node each incoming wavelength can be translated to any outgoing wavelength, this is often called the Virtual Wavelength Path or VWP concept). Without wavelength conversion (often called Wavelength Path or WP concept), the functionality can be compared to TDM networks without time-slot interchange (the time slot respective the wavelength of a connection is fixed throughout the network). Any intermediate wavelength conversion concept results in internal blocking in the nodes.

However, photonic networks provide also several particularities compared to classical networks. The exploitation of the wavelength (or frequency) domain provides a new degree of freedom for routing decisions. This leads to significant enhancement of routing possibilities: in every node a decision is made which link, fibre, and wavelength is chosen for the next section. On the other hand, the analog nature of optical networks results in new restrictions concerning for example maximum path lengths or a maximum number of nodes which can be crossed before electrical signal regeneration is required. Furthermore, the impact of blocking in the optical domain is much higher than in higher network layers due to the huge transport streams carried in the optical domain. 


\section{MODELLING AND TOOL REALISATION}

An important aspect for photonic network evaluation under dynamic traffic is how to model the random behaviour of wavelength channel setups/releases. We use two different modelling approaches depending on whether the focus is on the node architecture or on the mechanisms concerning the network as a whole.

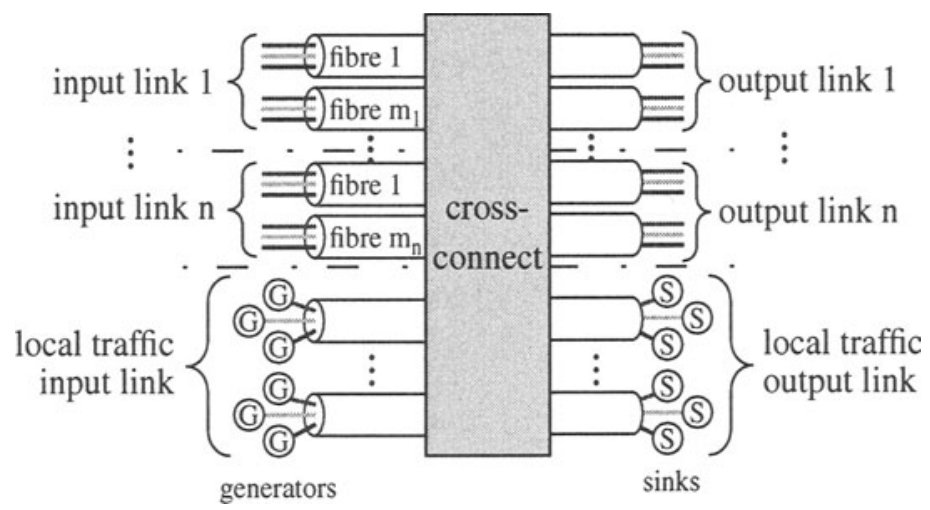

Figure 1 Modelling approach for the evaluation of node architectures

The approach that seems to be appropriate when investigating and comparing different node architectures under dynamic load scenarios uses one traffic generator for each wavelength (see Figure 1). In this case, for each source (i.e. a wavelength channel that is originating at a node) one generator is used. The source behaviour is specified by the distribution of the idle time of the represented source and the holding time of a connection (Figure 2). If a setup request has to be refused due to blocking in the node the source may be blocked for some time. After this period the call attempt is repeated after the repeat time. The distributions of the blocking and the repeat time can also be defined.

This source model is in contrast to most analytical approaches for evaluating node architectures described in literature, which use Poisson traffic with negative-exponentially distributed interarrival times and holding times ("pure chance traffic") [2]. Usually however, this simplified model holds only for a quasi infinite number of sources and is not a very good approximation for a smaller number of sources as expected in photonic networks. Especially, we expect the repeat behaviour of a wavelength channel to be different from "classical" sources due to the high traffic streams transported on a single wavelength. Dividing the time between a refused call attempt and the repetition of this call attempt into two phases has an additional advantage (besides the possibility of exactly modelling a real source). If the distribution types for holding and blocking time are chosen identically, the total offered load does not depend on the blocking values and therefore a comparison with analytical results can be done more easy. 


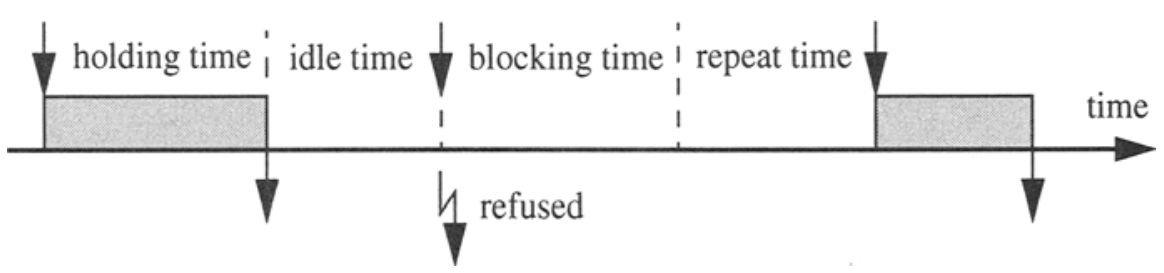

Figure 2 Source model used for node architecture evaluation

Apart from the local traffic input links, our model considers additional input links at the node which represent the traffic coming from other nodes. In this way it is also possible to build up an arbitrary concatenation of multiple nodes. Moreover, the architecture of the cross-connect defines its functionality and therefore determines the modelling of the "black box" shown in Figure 1. When modelling this part, aspects like the availability of wavelength conversion or the functionality of the applied switching stage inside the node have to be considered.

For larger networks with many channels per link however, this approach leads to a high number of generators in the simulation model. Therefore, for investigating network aspects and especially the influence of different routing strategies on network performance a different approach is applied. This approach uses one traffic generator to characterise the traffic between one pair of source and sink with appropriate distribution functions for the interarrival times of call requests and the call holding time, respectively (Figure 3). For the parameterization of the generators it is also possible to use measurement data.

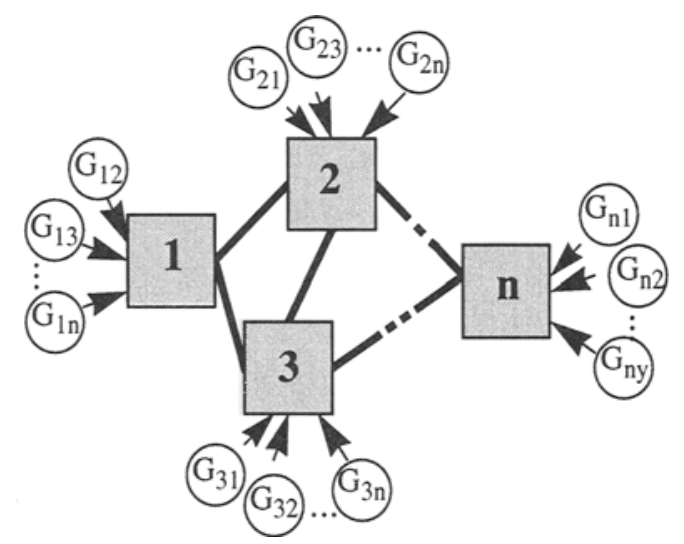

Figure 3 Modelling approach for investigating routing strategies

For the modelling of routing methods we also use two different modelling approaches:

- For hop-by-hop routing methods, each node has a separate routing table which contains the alternatives for the next hop. The decisions for the next path section are made locally in every node depending on the destination node. If a node can- 
not find an alternative, the call is either blocked or control is given back to the predecessor node (crankback).

- For source routing methods, each source contains a table with the full routing information for the path to any destination node. All decisions concerning the route choice are made only in the source node. This approach also covers all methods based on decisions made by a central network management (for example TMN).

The described modelling approaches form the base for our discrete event simulation tools which are realised in an object-oriented way in $\mathrm{C}++$. The tools allow a flexible definition of traffic characteristics. The topology of the investigated network can be chosen arbitrarily. This includes the placement and interconnection of nodes as well as the dimensioning of link capacities in terms of numbers of fibres. The routing strategy is described with an input parameter. If available, the routing alternatives can be defined freely. For the node architecture, several possibilities are already implemented, including the two extreme cases nodes without converters and nodes fully equipped with converters. Many other concepts can be thought of and are left for further studies.

The simulation tools provide a variety of results. Examples are link load or blocking probabilities for every link, every node, every source-destination node pair, or the whole network. The results are available as mean values with confidence intervals or - where appropriate - as distribution histograms.

\section{RESULTS AND CASE STUDIES}

\subsection{Evaluation of node architectures under dynamic traffic load}

The investigation of more complex node architectures usually requires simulative investigation methods. In the following, simulation results are presented for a CC with 8 input and 8 output links (with one or two fibres) including links for local traffic. Each output link is chosen with the same probability, i.e., no specific routing strategy is considered. We assume a share-per-node architecture where the available converters can be shared by all connections. The main goals are to evaluate the influence of the number of converters, of available WDM channels per fibre, and of fibres per link on the node performance, respectively. The offered load per wavelength and link is kept constant ( 0.5 Erlang), i.e., the total load is 32 Erlang for 8 wavelengths per fibre $(8 \mathrm{WL})$, or 64 Erlang for 16 wavelengths per fibre in the case of one fibre per link (in case of two fibres per link these values are already achieved with 4 or 16 wavelengths, respectively).

First, the results confirm the effect that already some converters improve the node performance significantly whereas from a certain point on further converters cannot decrease blocking any more (the lines are horizontal in Figure 4). Moreover, the well-known effect of "economy of scales" can also be seen here: with a higher total number of wavelength channels lower blocking values are achievable. However, the total number of converters required to achieve the minimum blocking is higher 


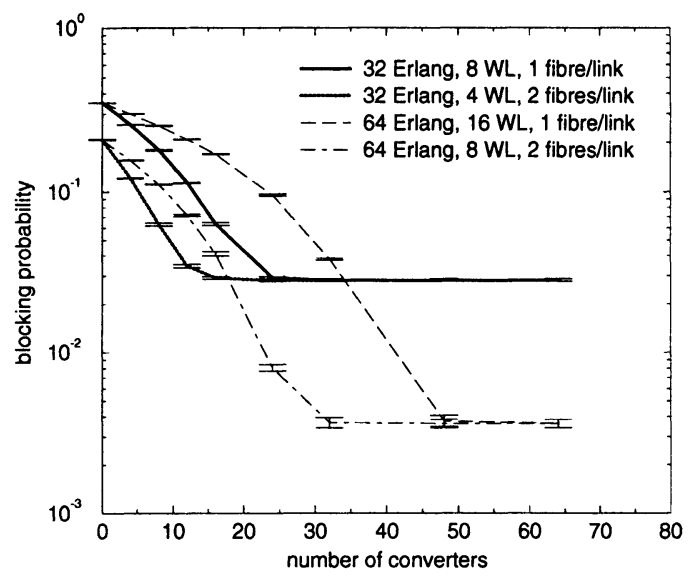

Figure 4 Blocking probability for a node with 8 input and 8 output links

than for smaller systems. Another possibility to improve the performance is to use multiple fibres - each with fewer wavelengths - for each link. In that case, fewer converters are required to decrease blocking significantly.

Other investigations showed that the quantitative performance improvement also strongly depends on the offered traffic load. With higher load, only small improvements can be achieved because then blocking due to overload is the main reason. Looking at the absolute blocking values, we see that for the assumed traffic dynamics blocking is relatively high. This leads to the conclusion that for optical crossconnecting we need either relatively high over-dimensioning, or the traffic dynamics have to be very small, or we have to reach larger bundles, i.e. bigger systems where blocking is improved due to economy of scales.

\subsection{Evaluation of routing strategies}

We concentrate in the following on the WP and the VWP case. The results are derived for an example network with nine nodes which could represent a national German network. The traffic demands (called static traffic demand in the following) were derived from population size and node distances applying simple rules. The network is dimensioned according to these static traffic demands using the shortest paths and then investigated under dynamic traffic conditions. Although our tools could also handle uni-directional calls, we do not focus on this and use only symmetrical bi-directional calls. Moreover, negative-exponential distributions are used for interarrival and call holding times.

In Figure 5, several routing methods are compared for the WP case. The first method is source routing based on the paths determined during network dimensioning (here: shortest paths) without alternatives. The other methods are hop-by-hop methods: with OOC (Originating-Office Control) only the originating node is able 
to choose an alternative path whereas all other nodes have to choose the first routing possibility towards the end node. In our case this means that each node checks all available wavelength channels for the primary direction. With SOC (SequentialOffice Control), each node is able to choose between a number of alternatives (i.e., all wavelength channels in multiple directions), but if a node is not able to find any way for the next path section the call is blocked. If SOC with crankback is used, then in case of blocking routing control may be returned to the predecessor which can now try to find other alternatives. Therefore, this is the most powerful method of the considered ones whereas fixed source routing is the most restricted one.

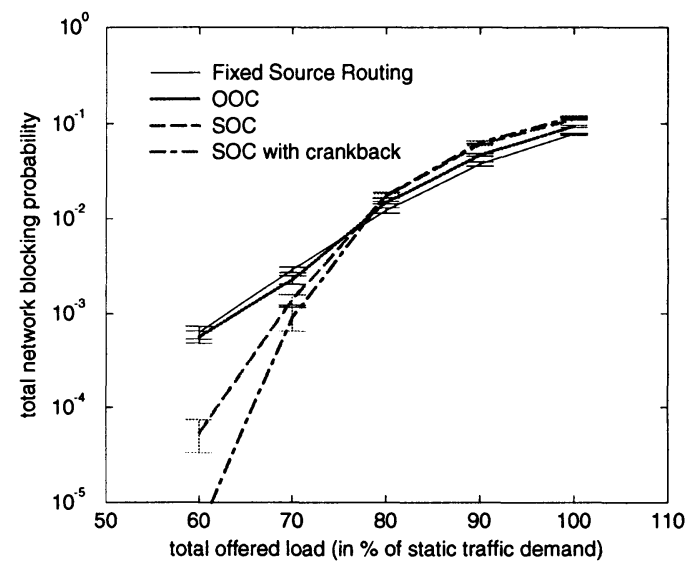

Figure 5 Comparison of routing methods in a WP network (no converters)

Figure 5 shows that the behaviour of the methods depends on the load. While for low load the methods which are able to choose between many alternatives lead to lower blocking probabilities, for high load the fixed source routing method which allows no alternatives is best. The reason is that alternatives usually lead to longer paths and therefore require more network resources. So alternate routing only leads to better overall performance if blocking occurs seldom. This effect is increased in the WP case considered here where a continuously free wavelength from source to destination has to be found.

Figure 6 compares two different routing strategies for WP and VWP respectively. Again, we see the differences between low and high load. Furthermore, the figure shows that wavelength converters are especially useful with sophisticated routing strategies: the improvement for SOC with crankback compared to fixed routing is much better in the VWP case than in the WP case.

Another result which can be derived from these figures is that a network planned for a certain static traffic capacity is not able to handle this traffic under dynamic changes, too. If dynamic traffic is present, the mean value of offered load has to be significantly lower than the static traffic demand to achieve acceptable blocking probabilities. 


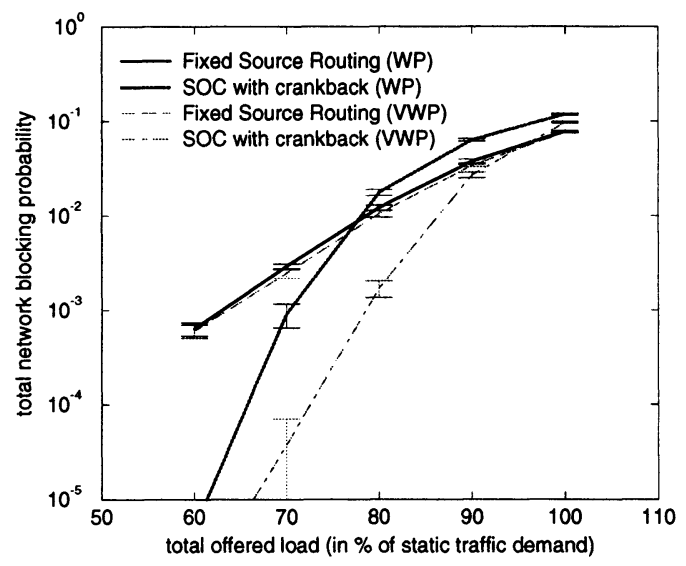

Figure 6 Comparison of different routing methods for WP and VWP case

\section{CONCLUSIONS AND OUTLOOK}

In this paper, we presented two modelling approaches for the simulative investigation of network performance under dynamic traffic conditions in an optical transport network. The developed tools based on these models allow performance investigations for arbitrary node and network structures for different traffic types. First results were derived for a single node showing the influence of the number of converters, the number of fibres, as well as the number of WDM channels per fibre on the node performance. Moreover, several routing strategies and their influence on network performance were evaluated.

Future work will focus on applying enhanced traffic models and on modelling the node architecture in more detail. Moreover, multi-layer aspects should be taken into account since the traffic streams in the optical layer result from aggregating various higher layer traffic streams. Finally, other routing methods will be evaluated considering more the specific features of an optical layer.

\section{ACKNOWLEDGEMENT}

This work has been supported by the German BMBF (Bundesministerium für Bildung, Wissenschaft, Forschung und Technologie) under contract 01BP504/2. The authors thank D. Porbadnigk and M. Koch for implementing the simulation tools.

\section{REFERENCES}

[1] A. Girard: Routing and Dimensioning in Circuit-Switched Networks; AddisonWesley, 1990

[2] J. Späth et al., Architecture and Performance Evaluation of Future Photonic Networks, Proceedings of the 12th ICCC, Seoul, 1995, Vol. 2, pp. 661 - 666 